\title{
OPTIMAL STOPPING WITH ADVANCED INFORMATION FLOW: SELECTED EXAMPLES
}

\author{
YAOZHONG HU \\ Department of Mathematics, University of Kansas \\ 405 Snow Hall, Lawrence, Kansas 66045-2142, U.S.A., and \\ Center of Mathematics for Applications (CMA) \\ Department of Mathematics, University of Oslo \\ Box 1053, Blindern, N-0316, Oslo, Norway \\ E-mail: hu@math.ku.edu \\ BERNT ØKSENDAL \\ Center of Mathematics for Applications (CMA) \\ Department of Mathematics, University of Oslo \\ Box 1053, Blindern, N-0316, Oslo, Norway, and \\ Norwegian School of Economics and Business Administration \\ Helleveien 30, N-5045, Bergen, Norway \\ E-mail: oksendal@math.uio.no
}

\begin{abstract}
We study optimal stopping problems for some functionals of Brownian motion in the case when the decision whether or not to stop before (or at) time $t$ is allowed to be based on the $\delta$-advanced information $\mathcal{F}_{t+\delta}$, where $\mathcal{F}_{s}$ is the $\sigma$-algebra generated by Brownian motion up to time $s, s \geq-\delta, \delta>0$ being a fixed constant. Our approach involves the forward integral and the Malliavin calculus for Brownian motion.
\end{abstract}

1. Introduction. The purpose of this paper is to study optimal stopping problems where the stopping time is allowed to depend on a larger filtration than the filtration of the underlying process. For example, the person who decides when to stop the system can base her decision on some future event, in addition to the history so far of the system.

More precisely, fix $\delta>0$ and let $B_{t}=B_{t}(\omega), t \in[-\delta, \infty), \omega \in \Omega$ be a standard Brownian motion on a probability space $(\Omega, \mathcal{F}, P)$, with $B_{-\delta}=0$. Define, for $t>-\delta$,

2000 Mathematics Subject Classification: Primary 93E20, 60H05, 60G51; Secondary 91B28.

Key words and phrases: optimal stopping, $\delta$-advanced information, forward integral, Malliavin calculus.

$\mathrm{Hu}$ is supported in part by the National Science Foundation under Grant No. DMS0504783.

The paper is in final form and no version of it will be published elsewhere. 


$$
\mathcal{F}_{t}=\sigma\left(B_{s},-\delta \leq s \leq t\right)
$$

i.e. $\mathcal{F}_{t}$ is the $\sigma$-algebra generated by the random variables $B_{s}(\cdot),-\delta \leq s \leq t$. Let $Y_{t}$ be an Itô diffusion in $\mathbb{R}^{k}$ given by

$$
\left\{\begin{array}{l}
d Y_{t}=b\left(Y_{t}\right) d t+\sigma\left(Y_{t}\right) d B_{t}, \quad t \geq-\delta, \\
Y_{-\delta}=y \in \mathbb{R}^{k}
\end{array}\right.
$$

where $b: \mathbb{R}^{k} \rightarrow \mathbb{R}$ and $\sigma: \mathbb{R}^{k} \rightarrow \mathbb{R}$ are given functions such that (1.2) has a unique strong solution. Let $\mathbb{H}=\left\{\mathcal{H}_{t}\right\}_{t \geq-\delta}$ be another filtration such that

$$
\mathcal{F}_{t} \subset \mathcal{H}_{t} \quad \text { for all } t \geq-\delta,
$$

We say that a function $\beta: \Omega \rightarrow[-\delta, \infty)$ is a stopping time with respect to $\left\{\mathcal{H}_{t}\right\}$ if

$$
\{\omega ; \beta(\omega) \leq t\} \in \mathcal{H}_{t} \quad \text { for all } t \geq-\delta .
$$

Let $\mathcal{B}_{0}$ denote a given subset of the set $\mathcal{B}$ of all $\mathbb{H}$-stopping times $\beta$. Let $f: \mathbb{R}^{k} \rightarrow \mathbb{R}$, $g: \mathbb{R}^{k} \rightarrow \mathbb{R}$ be given functions such that

$$
\mathbb{E}^{y}\left[\int_{-\delta}^{\infty}|f(Y(t))| d t+|g(Y(\beta))|\right]<\infty \quad \text { for all } \beta \in \mathcal{B}_{0},
$$

where $\mathbb{E}^{y}$ denotes expectation with respect to $P$ when $Y(0)=y$. In this paper we study special cases of the following performance functional:

$$
J(\beta)=\mathbb{E}^{y}\left[\int_{-\delta}^{\beta} f(Y(t)) d t+g(Y(\beta))\right], \quad y \in \mathbb{R}^{k}, \beta \in \mathcal{B}_{0}
$$

(where we put $g(Y(\beta))=0$ if $\beta=\infty$ ).

The problem we are studying in this paper is of the following form:

Problem. Find $\beta^{*} \in \mathcal{B}_{0}$ such that

$$
\sup _{\beta \in \mathcal{B}_{0}} J(\beta)=J\left(\beta^{*}\right) .
$$

We call this an anticipative optimal stopping problem, because the decision whether or not to stop at or before time $t$ might depend on future events. Such a problem is motivated by examples of insider trading in finance. See e.g. [10], [1], [3] and the references therein.

REMARK 1.1. Since an $\mathcal{H}_{t}$-stopping time $\beta$ is not necessarily an $\mathcal{F}_{t}$-stopping time (we may have $\mathcal{F}_{t} \neq \mathcal{H}_{t}$ ), we need to explain what we mean by $Y_{\beta(\omega)}(\omega), \omega \in \Omega$, i.e. the evaluation of $Y_{t}$ at $t=\beta$. In view of the use of forward integrals in the study of insider trading in finance (see e.g. [1]), we choose to use the definition

$$
Y_{\beta}:=y+\int_{-\delta}^{\beta} b\left(Y_{t}\right) d t+\int_{-\delta}^{\infty} \chi_{[0, \beta]}(t) \sigma\left(Y_{t}\right) d^{-} B_{t},
$$

where $d^{-} B_{t}$ indicates that the integral is interpreted as a forward integral.

Definition 1.2 ([11]). The forward integral of an $\mathcal{F}$-measurable process $\phi(t, \omega)$ with respect to $B$ is defined by

$$
\int_{-\delta}^{T} \phi(t) d^{-} B(t)=\lim _{\varepsilon \rightarrow 0} \int_{-\delta}^{T} \phi(t) \frac{B(t+\varepsilon)-B(t)}{\varepsilon} d t
$$

provided that the limit (in probability) exists. 
The reason for the usefulness of the forward integral in applied anticipating stochastic calculus is the following result:

LEMma $1.3([1])$. Suppose $\phi$ is forward integrable and càglàd (left continuous with right limits). Then

$$
\int_{-\delta}^{T} \phi(t) d^{-} B(t)=\lim _{\max \left|\Delta t_{i}\right| \rightarrow 0} \sum_{i} \phi\left(t_{i}\right)\left(B\left(t_{i+1}\right)-B\left(t_{i}\right)\right)
$$

(limit in probability) where $0=t_{0}<t_{1}<\cdots<t_{N}=T$ denotes partitions of $[-\delta, T]$.

In other words, the forward integral is the limit of Riemann sums where the integrand is evaluated at the left end points of the subintervals.

An Itô formula for forward integrals was established in [12]. See also [13].

The following definition will be useful (see [3], Definition 9.14). Here, and in the rest of the paper, $D_{t} F$ will denote the Malliavin derivative at $t$ of a random variable $F$. (See e.g. [3] for more information.)

Definition 1.4. Let $\mathbb{D}_{0}$ consist of all measurable processes $\phi$ such that

(i) $\phi$ is càglàd.

(ii) $\phi(t)$ is Malliavin differentiable for each $t \in[-\delta, T]$.

(iii) The paths $t \rightarrow D_{s} \phi(t)$ are càglàd for almost all $s \in[-\delta, T]$ and $\omega \in \Omega$.

(iv) The limit $D_{t+} \phi(t)=\lim _{s \rightarrow t^{+}} D_{s} \phi(t)$ exists with convergence in $L^{2}(P)$.

(v) $\phi$ is Skorohod integrable.

In our paper we will need the following result, the first version of which was proved in $[11]$.

Lemma $1.5([11])$. Let $\phi \in \mathbb{D}_{0}$. Then

$$
\mathbb{E}\left[\int_{-\delta}^{T} \phi(t) d^{-} B(t)\right]=\mathbb{E}\left[\int_{-\delta}^{T} D_{t+} \phi(t) d(t)\right] .
$$

The solution of Problem A appears to be very difficult, even in the simplest cases. In this paper we will restrict ourselves to the following special cases:

$$
\begin{aligned}
\mathcal{H}_{t} & =\mathcal{F}_{t+\delta}, \quad t \geq-\delta, \\
Y_{t} & =\left(t, B_{t}\right), \quad \text { where } B_{t} \text { is a } 1 \text {-dimensional Brownian motion, } \\
\mathcal{B}_{0} & =\mathcal{B}_{0}(\delta)
\end{aligned}
$$

where $\mathcal{B}_{0}(\delta)$ is the set of $\mathcal{F}_{t+\delta}$-stopping times $\beta$ which are of the first exit type, i.e. of the form $\beta=\beta_{a}=\inf \left\{t \geq-\delta ; B_{t+\delta} \geq a\right\} \wedge T$ for some constants $a \in \mathbb{R}, T \in(0, \infty]$. Similarly we let $\mathcal{B}(\delta)$ denote the set of all $\mathcal{F}_{t+\delta}$-stopping times $\beta: \Omega \rightarrow[-\delta, \infty]$.

We call such stopping times $\delta$-advanced, or stopping times with $\delta$-advanced information.

REMARK 1.6. Note that for any random time $\beta: \Omega \rightarrow[-\delta, \infty]$ we have that $\{\omega ; \beta(\omega)$ $\leq t\} \in \mathcal{F}_{t+\delta}$ for all $t \geq 0$ if and only if $\{\omega ; \beta(\omega)+\delta \leq t\} \in \mathcal{F}_{t}$ for all $t \geq 0$. Hence $\beta$ is an $\mathcal{F}_{t+\delta}$-stopping time if and only if $\tau:=\beta+\delta$ is an $\mathcal{F}_{t}$-stopping time. 
Equivalently, all $\mathcal{F}_{t+\delta}$-stopping times $\beta$ may be written $\beta=\tau-\delta$, where $\tau: \Omega \rightarrow[0, \infty]$ is an $\mathcal{F}_{t}$-stopping time.

In Section 3 we will study the anticipative optimal stopping problem where

$$
J(\beta)=J_{1}(\beta)=\mathbb{E}\left[e^{-\rho \beta}\left(B_{\beta}-c\right)\right], \quad \beta \in \mathcal{B}_{0}(\delta) .
$$

where $\rho>0, c \geq 0$ are constants.

In Section 4 we want to minimize

$$
J(\beta)=J_{2}(\beta)=\mathbb{E}\left[\int_{-\delta}^{\beta} e^{-\rho t} B_{t} d t\right], \quad \beta \in \mathcal{B}_{0}(\delta) .
$$

We give an implicit solution for the case (1.15) and an explicit solution for the case (1.16). In particular, our solution of case (1.16) implies that

$$
\inf _{\beta \in \mathcal{B}(\delta)} J_{2}(\beta)<\inf _{\beta \in \mathcal{B}_{0}(\delta)} J_{2}(\beta) .
$$

Thus $\left(\delta\right.$-advanced) first exit times $\beta_{a}$ are not always optimal for $\delta$-advanced optimal stopping problems. This is in contrast to generic classical optimal stopping problems for Itô diffusions. (See e.g. [7], Chapter 10.)

2. The case when there is no cost and no discount. In this section we shall

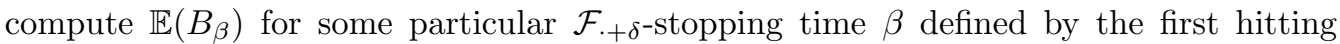
time to a half line and we also introduce some notations and methods.

As before let $\left(B_{t}, t \geq 0\right)$ be a Brownian motion on a probability space $(\Omega, \mathcal{F}, P)$ and let $\mathcal{F}_{t}, t \geq 0$, be the natural filtration associated with this Brownian motion. Fix $T>0$.

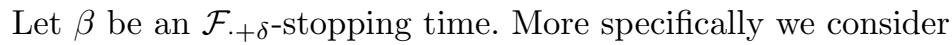

$$
\beta=\inf \left\{t>-\delta ; B_{t+\delta} \geq a\right\} \wedge T
$$

for some fixed $a \in \mathbb{R}$. We first compute $\mathbb{E}\left[B_{\beta}\right]$ in this section and $\mathbb{E}\left[e^{-\rho \beta}\left(B_{\beta}-c\right)\right]$ will be computed in the next section.

Denote

$$
M_{t}=\max _{0 \leq s \leq t} B_{s} \quad \text { and } \quad \theta_{t}=\arg \max _{0 \leq s \leq t} B_{s},
$$

the unique time such that $B_{\theta_{t}}=\max _{0 \leq s \leq t} B_{s}$.

Since $\beta>s$ is equivalent to $M_{s+\delta}<a$, we have $\chi_{[0, \beta)}(s)=\chi_{(-\infty, a)}\left(M_{s+\delta}\right)$. Thus

$$
D_{s+} \chi_{[0, \beta)}(s)=D_{s+} \chi_{(-\infty, a)}\left(M_{s+\delta}\right)=-\delta_{a}\left(M_{s+\delta}\right) D_{s+} M_{s+\delta}=-\delta_{a}\left(M_{s+\delta}\right) \chi_{\left[0, \theta_{s+\delta}\right]}(s) .
$$

Thus we have, by Lemma 1.5,

$$
\mathbb{E} \int_{-\delta}^{\beta} \phi(s) d^{-} B_{s}=-\int_{0}^{T} \mathbb{E}\left[\phi(s) \delta_{a}\left(M_{s+\delta}\right) \chi_{\left[0, \theta_{s+\delta}\right]}(s)\right] d s .
$$

In the case that $\phi(s)=1$, that is, we compute $\mathbb{E}\left(B_{\beta}\right)$, we have

$$
\mathbb{E}\left(B_{\beta}\right)=-\int_{0}^{T} \mathbb{E}\left\{\delta_{a}\left(M_{s+\delta}\right) \chi_{\left[0, \theta_{s+\delta}\right]}(s)\right\} d s .
$$

Now the joint pdf of $M_{t}$ and $\theta_{t}$ is (see [2], p. 209, Formula 1.13.4)

$$
P\left(M_{t} \in d y, \theta_{t} \in d v\right)=\frac{y e^{-\frac{y^{2}}{2 v}}}{\pi \sqrt{(t-v) v^{3}}} d v d y \text {. }
$$


Thus, the joint pdf of $M_{s+\delta}$ and $\theta_{s+\delta}$ is

$$
q_{s, \delta}(v, y)=\frac{y e^{-\frac{y^{2}}{2 v}}}{\pi \sqrt{(s+\delta-v) v^{3}}} .
$$

Therefore we have

$$
\begin{aligned}
\mathbb{E}\left(B_{\beta}\right) & =\int_{0}^{T} \int_{\mathbb{R}} \int_{0}^{s+\delta} \delta(y-a) \chi_{[s, s+\delta]}(v) q_{s, \delta}(v, y) d y d v d s \\
& =\int_{0}^{T} \int_{0}^{s+\delta} \chi_{[s, s+\delta]}(v) q_{s, \delta}(v, a) d v d s=\int_{0}^{T} \int_{v-\delta}^{v} q_{s, \delta}(v, a) d s d v=\frac{\sqrt{\delta}}{\pi} \int_{\frac{a^{2}}{2 T}}^{\infty} \frac{e^{-u}}{\sqrt{u}} d u .
\end{aligned}
$$

Theorem 2.1. Let $\tilde{\beta}=\inf \left\{t>0 ; B_{t+\delta} \geq a\right\}$ and $\beta=\tilde{\beta} \wedge T$. Then for any $\delta>0$ and $a>0$, we have

$$
\mathbb{E}\left(B_{\beta}\right)=\frac{\sqrt{\delta}}{\pi} \int_{\frac{a^{2}}{2 T}}^{\infty} \frac{e^{-u}}{\sqrt{u}} d u
$$

It is clear from this computation that when $\delta=0, \mathbb{E}\left(B_{\beta}\right)=0$ and for fixed $\delta>0$, the maximum is attained when $a=0$.

3. Optimal stopping for $\mathbb{E}\left[e^{-\rho \beta}\left(B_{\beta}-c\right)\right]$. The computation of $\mathbb{E}\left[e^{-\rho \beta}\left(B_{\beta}-c\right)\right]$ is slightly more complicated. First we compute $\mathbb{E}\left[e^{-\rho \beta}\right]$. We need to know the distribution of $\beta$. From [2], p. 197, Formula (1.1.4),

$$
P(\beta>\xi)=P\left(M_{\xi+\delta}<a\right)=1-P\left(\sup _{0 \leq s \leq \xi+\delta} B_{s} \geq a\right)=1-q_{1, s, \mu, \delta, a}(\xi)
$$

where

$$
q_{1, s, \mu, \delta, a}(\xi)=\frac{1}{2} \operatorname{Erfc}\left(\frac{a}{\sqrt{2(\xi+\delta)}}-\frac{\mu \sqrt{\xi+\delta}}{\sqrt{2}}\right)+\frac{1}{2} e^{2 \mu a} \operatorname{Erfc}\left(\frac{a}{\sqrt{2(\xi+\delta)}}+\frac{\mu \sqrt{\xi+\delta)}}{\sqrt{2}}\right)
$$

with $\operatorname{Erfc}(x)=\frac{1}{\sqrt{2 \pi}} \int_{-\infty}^{x} e^{-t^{2} / 2} d t$ being the error function. Now $\mu=0$. Hence

$$
P(\beta>\xi)=\int_{\frac{a}{\sqrt{2(\xi+\delta)}}}^{\infty} e^{-\frac{x^{2}}{2}} d x .
$$

Therefore

$$
\begin{aligned}
1-\mathbb{E}\left[e^{-\rho \beta}\right] & =\rho \int_{0}^{\beta} e^{-\rho \xi} d \xi=\rho \mathbb{E} \int_{0}^{T} e^{-\rho \xi} \mathbb{E} \chi_{\{\beta>\xi\}} d \xi=\rho \int_{0}^{T} e^{-\rho \xi} \int_{\frac{a}{\sqrt{2(\xi+\delta)}}}^{\infty} e^{-\frac{x^{2}}{2}} d x d \xi \\
& =\rho \int_{\frac{a}{\sqrt{2(T+\delta)}}}^{\infty} \int_{\frac{a^{2}}{2 x^{2}}-\delta}^{T} e^{-\rho \xi} d \xi e^{-\frac{x^{2}}{2}} d x=\int_{\frac{a}{\sqrt{2(T+\delta)}}}^{\infty}\left[e^{\rho \delta-\frac{\rho a^{2}}{2 x^{2}}}-e^{-\rho T}\right] e^{-\frac{x^{2}}{2}} d x .
\end{aligned}
$$

This is summarized in

Proposition 3.1. Let $\rho$ be a given real number. Let $\beta$ be an $\mathcal{F}_{-+\delta}$-stopping time. More specifically we consider

$$
\beta=\inf \left\{t>-\delta ; B_{t+\delta} \geq a\right\} \wedge T
$$


for some fixed $a \in \mathbb{R}$. Then

$$
\mathbb{E}\left[e^{-\rho \beta}\right]=1-\int_{\frac{a}{\sqrt{2(T+\delta)}}}^{\infty}\left[e^{\rho \delta-\frac{\rho a^{2}}{2 x^{2}}}-e^{-\rho T}\right] e^{-\frac{x^{2}}{2}} d x .
$$

Letting $T \rightarrow \infty$, we have

Corollary 3.2. Let $\rho>0$ be a given positive real number. Let $\beta$ be an $\mathcal{F}_{+\delta^{-}}$-stopping time. More specifically we consider

$$
\beta=\inf \left\{t>-\delta ; B_{t+\delta} \geq a\right\}
$$

for some fixed $a \in \mathbb{R}$. Then

$$
\mathbb{E}\left[e^{-\rho \beta}\right]=1-\int_{0}^{\infty} e^{\rho \delta-\frac{\rho a^{2}}{2 x^{2}}-\frac{x^{2}}{2}} d x .
$$

The computation of $\mathbb{E}\left[e^{-\rho \beta} B_{\beta}\right]$ is more sophisticated and a more clever technique is needed. We have

$$
\begin{aligned}
1-\mathbb{E}\left[e^{-\rho \beta} B_{\beta}\right] & =\rho \mathbb{E}\left[\int_{0}^{T} e^{-\rho u} \chi_{\{u \leq \beta\}} d u B_{\beta}\right]=\rho \mathbb{E}\left[\int_{-\delta}^{T} \int_{0}^{T} e^{-\rho u} \chi_{\{u \leq \beta\}} \chi_{\{s \leq \beta\}} d u d B_{s}\right] \\
& =\rho \mathbb{E}\left[\int_{-\delta}^{T} \int_{0}^{T} e^{-\rho u} \chi_{\{\beta \geq u \vee s\}} d u d B_{s}\right] \\
& =\rho \mathbb{E}\left[\int_{0}^{T} \int_{0}^{T} e^{-\rho u} \chi_{(-\infty, a)}\left(M_{u \vee s+\delta}\right) d u d B_{s}\right] \\
& =\rho \int_{0}^{T} \int_{0}^{T} e^{-\rho u} \mathbb{E}\left[D_{s+\chi_{(-\infty, a)}}\left(M_{u \vee s+\delta}\right)\right] d u d s \\
& =\rho \int_{0}^{T} \int_{0}^{T} e^{-\rho u} \mathbb{E}\left[\delta_{a}\left(M_{u \vee s+\delta}\right) \chi_{\left[0, \theta_{u \vee s+\delta}\right]}\right] d u d s .
\end{aligned}
$$

The joint pdf of $M_{u \vee s+\delta}$ and $\theta_{u \vee s+\delta}$ is

$$
\tilde{q}_{s, u, \delta}(v, y)=\frac{y e^{-\frac{y^{2}}{2 v}}}{\pi \sqrt{(u \vee s+\delta-v) v^{3}}}, \quad v<u \vee s+\delta .
$$

Therefore

$$
\begin{aligned}
1-\mathbb{E}\left[e^{-\rho \beta} B_{\beta}\right]= & \rho \int_{0}^{T} \int_{0}^{T} \int_{0}^{T} \int_{0}^{T} e^{-\rho u} \delta_{a}(y) \chi_{[0, v]}(s) \tilde{q}_{s, u, \delta}(v, y) d y d v d u d s \\
= & \rho \int_{0}^{T} \int_{0}^{T} \int_{0}^{T} e^{-\rho u} \chi_{[0, v]}(s) \tilde{q}_{s, u, \delta}(v, a) d v d u d s \\
= & \rho \iiint_{\{s<u, s<u, v<u+\delta\}} e^{-\rho u} \frac{a e^{-\frac{a^{2}}{2 v}}}{\pi \sqrt{(u+\delta-v) v^{3}}} d s d v d u \\
& +\rho \iiint_{\{s<u, s>u, v<u+\delta\}} e^{-\rho u} \frac{a e^{-\frac{a^{2}}{2 v}}}{\pi \sqrt{(s+\delta-v) v^{3}}} d s d v d u
\end{aligned}
$$




$$
\begin{aligned}
& =\frac{a \rho}{\pi} \iint_{\{v<u+\delta\}} \frac{e^{-\rho u-\frac{a^{2}}{2 v}}}{\sqrt{(u+\delta-v) v^{3}}} d v d u \\
& \quad+\frac{2 a \rho}{\pi} \iint_{\{u<v\}}[\sqrt{\delta}-\sqrt{u \vee(v-\delta)+\delta-v}] e^{-\rho u-\frac{a^{2}}{2 v}} d v d u .
\end{aligned}
$$

Thus we have

THEOREM 3.3. Let $\delta>0, \rho$, and $c$, be given real numbers. Let $\beta$ be an $\mathcal{F}_{++\delta \text {-stopping }}$ time. More specifically we consider

$$
\beta=\inf \left\{t>-\delta ; B_{t+\delta} \geq a\right\}
$$

for some fixed $a \in \mathbb{R}$. Then

$$
\begin{aligned}
\mathbb{E}\left[e^{-\rho \beta}\left(B_{\beta}-c\right)\right]=1 & +c-\frac{a \rho}{\pi} \iint_{\{v<u+\delta\}} \frac{e^{-\rho u-\frac{a^{2}}{2 v}}}{\sqrt{(u+\delta-v) v^{3}}} d v d u \\
& -\frac{2 a \rho}{\pi} \iint_{\{u<v\}}[\sqrt{\delta}-\sqrt{u \vee(v-\delta)+\delta-v}] e^{-\rho u-\frac{a^{2}}{2 v}} d v d u \\
& -c \int_{\frac{a}{\sqrt{2(T+\delta)}}}^{\infty}\left[e^{\rho \delta-\frac{\rho a^{2}}{2 x^{2}}}-e^{-\rho T}\right] e^{-\frac{x^{2}}{2}} d x .
\end{aligned}
$$

It is clear from the above theorem that $\mathbb{E}\left[e^{-\rho \beta}\left(B_{\beta}-c\right)\right]$ is a continuous function of $a$ for on any compact set of $a$. Thus it has maximum on any compact set of $a$. Numerical computation is needed to find the maximum.

4. Optimal stopping for $\mathbb{E}\left[\int_{0}^{\beta} e^{-\rho t} B_{t} d t\right]$. Now consider the problem to find an $\mathcal{F}_{\cdot+\delta^{-}}$ stopping time $\tau$ which maximizes

$$
\mathbb{E}\left[\int_{0}^{\tau} e^{-\rho t} B_{t} d t\right]
$$

We consider stopping times of the form

$$
\beta=\inf \left\{t>-\delta, B_{t} \geq a\right\}
$$

where $a>0$. We use

$$
\mathbb{E}\left[\int_{-\delta}^{\beta} e^{-\rho t} B_{t} d t\right]=\int_{-\delta}^{\infty} e^{-\rho t} \mathbb{E}\left[\chi_{\{\beta>t\}} B_{t}\right] d t .
$$

Denote again $M_{t}=\sup _{0 \leq r \leq t} B_{r}$. Now

$$
\mathbb{E}\left[\chi_{\{\beta>t\}} B_{t}\right]=\int_{0}^{t} \mathbb{E}\left[D_{s} \chi_{\{\beta>t\}}\right] d s=\int_{0}^{t} \mathbb{E}\left[D_{s} \chi_{\left\{M_{t}<a\right\}}\right] d s=-\int_{0}^{t} \mathbb{E}\left[\delta_{a}\left(M_{t}\right) \chi_{\left\{s<\theta_{t}\right\}}\right] d s .
$$

Since the joint pdf of $M_{t}$ and $\theta_{t}$ is

$$
\tilde{q}_{t}(v, y)=\frac{y e^{-\frac{y^{2}}{2 v}}}{\pi \sqrt{(t+\delta-v) v^{3}}}, \quad v<t+\delta
$$


we have

$$
\begin{aligned}
\mathbb{E}\left[\chi_{\{\beta>t\}} B_{t}\right] & =-\int_{0}^{t} \iint \delta_{a}(y) \chi_{\{s<v\}} \frac{y e^{-\frac{y^{2}}{2 v}}}{\pi \sqrt{(t+\delta-v) v^{3}}} d s d v d y \\
& =-\iint_{0<s<v<t+\delta} \frac{a e^{-\frac{a^{2}}{2 v}}}{\pi \sqrt{(t+\delta-v) v^{3}}} d s d v \\
& =-\iint_{0<s<v<t+\delta} \frac{a e^{-\frac{a^{2}}{2 v}}}{\pi \sqrt{v} \sqrt{t+\delta-v}} d v
\end{aligned}
$$

Thus

$$
\begin{aligned}
\mathbb{E}\left[\int_{-\delta}^{\beta} e^{-\rho t} B_{t} d t\right] & =-\iint_{0<v<t+\delta<\infty} e^{-\rho t} \frac{a e^{-\frac{a^{2}}{2 v}}}{\pi \sqrt{v} \sqrt{t+\delta-v}} d s d v \\
& =-\frac{a g(\rho, \delta)}{\pi} \int_{0}^{\infty} e^{-\rho v-\frac{a^{2}}{2 v}} v^{-1 / 2} d v
\end{aligned}
$$

where

$$
g(\rho, \delta)=\int_{0}^{\infty} \frac{e^{-\rho s+\rho \delta}}{\sqrt{s}} d s=\Gamma\left(\frac{1}{2}\right) e^{\rho \delta} \rho^{-\frac{1}{2}}=\sqrt{\pi} e^{\rho \delta} \rho^{-\frac{1}{2}} .
$$

By [4], Formula 3.471 (9) we have

$$
\int_{0}^{\infty} e^{-\rho v-\frac{a^{2}}{2 v}} v^{-1 / 2} d v=2\left(\frac{a^{2}}{2 \rho}\right)^{1 / 4} K_{1 / 2}\left(2 \sqrt{\frac{a^{2} \rho}{2}}\right)
$$

and again by [4], Formula 8.469 (3) we have

$$
K_{1 / 2}\left(2 \sqrt{\frac{a^{2} \rho}{2}}\right)=\sqrt{\frac{\pi}{2 \cdot 2 \sqrt{\frac{a^{2} \rho}{2}}}} e^{-2 \sqrt{\frac{a^{2} \rho}{2}}} .
$$

Therefore

$$
\int_{0}^{\infty} e^{-\rho v-\frac{a^{2}}{2 v}} v^{-1 / 2} d v=\frac{\sqrt{\pi}}{\sqrt{\rho}} e^{-\sqrt{2 a^{2} \rho}}
$$

Then

$$
\mathbb{E}\left[\int_{-\delta}^{\beta} e^{-\rho t} B_{t} d t\right]=-\frac{a g(\rho, \delta)}{\pi} \frac{\sqrt{\pi}}{\sqrt{\rho}} e^{-\sqrt{2 a^{2} \rho}}=-\frac{a e^{\rho \delta} e^{-a \sqrt{2 \rho}}}{\rho} .
$$

From this, the minimum is easily seen to be attained at $a=\frac{1}{\sqrt{2 \rho}}$.

We have proved:

TheOREM 4.1.

$$
\inf _{\beta \in \mathcal{B}_{0}(\delta)} \mathbb{E}\left[\int_{-\delta}^{\beta} e^{-\rho t} B_{t} d t\right]=-\frac{e^{\rho \delta-1}}{\rho \sqrt{2 \rho}},
$$

and the minimum is attained at

$$
\beta=\beta_{0}=\inf \left\{t>-\delta ; B_{t+\delta} \geq a\right\}=\inf \left\{s>0 ; B_{s} \geq a\right\}-\delta
$$

where

$$
a=\frac{1}{\sqrt{2 \rho}} .
$$




\section{REMARK 4.2 .}

(i) Note that this threshold $a=\frac{1}{\sqrt{2 \rho}}$ does not depend on $\delta$. In fact, we get the same threshold for stopping when $\delta=0$, and even when there is a delay from the time when decision to stop is taken and the time when the system actually stops. See [8].

(ii) If $\delta=0$ the problem can be solved easily using variational inequalities, and this gives the value

$$
-\frac{e^{-1}}{\rho \sqrt{2 \rho}} \quad\left(\text { when } B_{0}=0\right)
$$

Comparing with (4.9) we see that the optimal $\mathcal{B}_{0}(\delta)$ performance improves by factor $e^{\rho \delta}$ when we go from $\delta=0$ to $\delta>0$. This ratio $e^{\rho \delta}$ is a measure of the value of the $\delta$-advanced information flow.

Next we show that the optimal $\delta$-advanced stopping of first exit type, $\beta_{0} \in \mathcal{B}_{0}(\delta)$ given by (4.10)-(4.11) cannot be optimal among all $\beta \in \mathcal{B}(\delta)$.

TheOREM 4.3. Let $\delta>0$. Then

$$
\inf _{\beta \in \mathcal{B}(\delta)} J_{2}(\beta)<\inf _{\beta \in \mathcal{B}_{0}(\delta)} J_{2}(\beta) .
$$

Proof. Put

$$
\tau_{0}=\inf \left\{s>0 ; B_{s} \geq 1 / \sqrt{2 \rho}\right\}
$$

Then

$$
\beta_{0}=\tau_{0}-\delta \quad \text { and } \quad B\left(\tau_{0}\right) \geq \frac{1}{\sqrt{2 \rho}} .
$$

Define

$$
\Omega_{0}=\left\{\omega \in \Omega ; B_{\beta_{0}}<0\right\}
$$

Then by basic properties of Brownian motion we have $P\left(\Omega_{0}\right)>0$. Define another $\mathcal{F}_{t+\delta^{-}}$ stopping time $\tilde{\beta}$ as follows:

$$
\tilde{\beta}(\omega)=\inf \left\{s \in\left[\tau_{0}-\delta, \tau_{0}\right] ; s \text { is a minimum point of } s \rightarrow \int_{\tau_{0}-\delta}^{s} e^{-\rho t} B_{t} d t\right\}, \omega \in \Omega .
$$

Then $\beta_{0} \leq \tilde{\beta}$ a.s. and $P\left[\beta_{0}<\tilde{\beta}\right]=P\left(\Omega_{0}\right)>0$. Moreover, $\int_{\beta_{0}}^{\tilde{\beta}} e^{-\rho t} B_{t} d t<0$ if $\omega \in \Omega_{0}$. Therefore

$$
\mathbb{E}\left[\int_{-\delta}^{\tilde{\beta}} e^{-\rho t} B_{t} d t\right]<\mathbb{E}\left[\int_{-\delta}^{\beta_{0}} e^{-\rho t} B_{t} d t\right],
$$

which proves Theorem 4.3.

REMARK 4.4. We conjecture that $\tilde{\beta}$ defined in (4.13) is an optimal $\mathcal{F}_{t+\delta}$-stopping time in $\mathcal{B}(\delta)$.

Acknowledgments. We are grateful to Christian Bender, Thorsten Schmidt and Jerzy Zabczyk for helpful comments.

\section{References}

[1] F. Biagini and B. Øksendal, A general stochastic calculus approach to insider trading, Appl. Math. Optim. 52 (2005), 167-181. 
[2] A. N. Borodin and P. Salminen, Handbook of Brownian Motion-Facts and Formulae, 2nd ed., Probability and its Applications, Birkhäuser Verlag, Basel, 2002.

[3] G. Di Nunno, B. Øksendal and F. Proske, Malliavin Calculus for Lévy Processes with Applications to Finance, Forthcoming book, to be published by Springer.

[4] I. S. Gradshteyn and I. M. Ryzhik, Tables of Integrals, Series, and Products, Academic Press, San Diego, CA, 2000.

[5] J. M. Harrison, Brownian Motion and Stochastic Flow Systems, Wiley, New York, 1985.

[6] D. Nualart, The Malliavin Calculus and Related Topics, Springer-Verlag, New York, 1995.

[7] B. Øksendal, Stochastic Differential Equations. An Introduction with Applications, 6th ed., Universitext, Springer-Verlag, Berlin, 2003.

[8] B. Øksendal, Optimal stopping with delayed information, Stoch. Dyn. 5 (2005), 271-280.

[9] B. Øksendal and A. Sulem, Applied Stochastic Control of Jump Diffusions, 2nd ed., Universitext, Springer-Verlag, Berlin, 2007.

[10] I. Pikovsky and I. Karatzas, Anticipative portfolio optimization, Adv. Appl. Probab. 28 (1996), 1095-1122.

[11] F. Russo and P. Vallois, Forward, backward and symmetric stochastic integration, Probab. Theory Related Fields 97 (1993), 403-421.

[12] F. Russo and P. Vallois, The generalized covariation process and Itô formula, Stochastic Process. Appl. 59 (1995), 81-104.

[13] F. Russo and P. Vallois, Stochastic calculus with respect to continuous finite quadratic variation processes, Stochastics Stochastics Rep. 70 (2000), 1-40. 